\title{
Genetic Analysis Workshop 15: gene expression analysis and approaches to detecting multiple functional loci
} Heather J Cordell*1, Mariza de Andrade ${ }^{2}$, Marie-Claude Babron³, Christopher W Bartlett ${ }^{4}$, Joseph Beyene ${ }^{5}$, Heike Bickeböller ${ }^{6}$, Robert Culverhouse ${ }^{7}$, L Adrienne Cupples ${ }^{8}$, E Warwick Daw ${ }^{9}$, Josée Dupuis8, Catherine T Falk ${ }^{10}$, Saurabh Ghosh ${ }^{11}$, Katrina A Goddard 12 , Ellen L Goode ${ }^{2}$, Elizabeth R Hauser ${ }^{13}$, Lisa J Martin ${ }^{14}$, Maria Martinez ${ }^{15}$, Kari E North ${ }^{16}$, Nancy L Saccone ${ }^{7}$, Silke Schmidtt ${ }^{13}$, William Tapper ${ }^{17}$, Duncan Thomas ${ }^{18}$, David Tritchler ${ }^{19}$, Veronica J Vieland ${ }^{4}$, Ellen M Wijsman ${ }^{20}$, Marsha A Wilcox ${ }^{21}$, John S Witte 22 , Qiong Yang 8 , Andreas Ziegler ${ }^{23}$, Laura Almasy ${ }^{24}$ and Jean W MacCluer ${ }^{24}$

Address: ${ }^{1}$ Institute of Human Genetics, Newcastle University, Newcastle upon Tyne NE1 3BZ, UK, ${ }^{2}$ Mayo Clinic, Rochester, Minnesota 55905, USA, ${ }^{3}$ INSERM U535, BP 1000, Villejuif, 94817, France, ${ }^{4}$ Columbus Children's Research Institute, Columbus, Ohio 43205, USA, ${ }^{5}$ Hospital for Sick Children, Toronto, ON M5G 1X8 Canada, ${ }^{6}$ Universität Göttingen, 37073, Germany, ${ }^{7}$ Washington University, St Louis, Missouri 63110, USA, ${ }^{8}$ Boston University School of Public Health, Boston, Massachusetts, USA, ${ }^{9}$ Washington University School of Medicine, Division of Statistical Genomics, St. Louis, Missouri 63108, USA, ${ }^{10}$ Teaneck, New Jersey 07666, USA, ${ }^{11}$ Indian Statistical Institute, Kolkata 700 108, India, ${ }^{12}$ Case Western Reserve University, Cleveland, Ohio 44106-7281, USA, ${ }^{13}$ Duke University, Durham, North Carolina 27710, USA, ${ }^{14}$ Cincinnati Children's Hospital, Cincinnati, Ohio 45229, USA, ${ }^{15}$ INSERM U563, Toulouse, 31024, France, ${ }^{16}$ University of North Carolina, Chapel Hill, North Carolina 27514, USA, ${ }^{17}$ University of Southampton, Hampshire SO166YD, UK, ${ }^{18}$ University of Southern California, Los Angeles, California 94720, USA, ${ }^{19}$ Ontario Cancer Institute, Toronto, Ontario, M5G 2M9 Canada, ${ }^{20}$ University of Washington, Seattle, Washington 98195-7720, USA, ${ }^{21}$ i3 Drug Safety, Waltham, Massachusetts 02451, USA, ${ }^{22}$ University of California, San Francisco, California 94143-0794, USA, ${ }^{23}$ Institute of Medical Biometry and Statistics, Lübeck, 23538, Germany and ${ }^{24}$ Southwest Foundation for Biomedical Research, San Antonio, Texas 78245, USA

Email: Heather J Cordell* - heather.cordell@newcastle.ac.uk; Mariza de Andrade - mandrade@mayo.edu; Marie-

Claude Babron - babron@vjf.inserm.fr; Christopher W Bartlett - bartletc@paediatrics.ohio-state.edu;

Joseph Beyene - joseph@utstat.toronto.edu; Heike Bickeböller - hbickeb@gwdg.de; Robert Culverhouse - rculverh@im.wustl.edu; L Adrienne Cupples - adrienne@bu.edu; E Warwick Daw - warwick@wustl.edu; Josée Dupuis - dupuis@bu.edu;

Catherine T Falk - cfalk@sci.ccny.cuny.edu; Saurabh Ghosh - saurabh@isical.ac.in; Katrina A Goddard - katrina@darwin.cwru.edu; Ellen L Goode - egoode@mayo.edu; Elizabeth R Hauser - bhauser@chg.duhs.duke.edu; Lisa J Martin - Lisa.Martin@chmcc.org; Maria Martinez - maria.martinez@toulouse.inserm.fr; Kari E North - kari_north@unc.edu; Nancy L Saccone - nlims@vodka.wustl.edu; Silke Schmidt - sschmidt@chg.duhs.duke.edu; William Tapper - wjt@soton.ac.uk; Duncan Thomas - dthomas@usc.edu; David Tritchler - tritchle@uhnres.utoronto.ca; Veronica J Vieland - vielandv@ccri.net; Ellen M Wijsman - wijsman@u.washington.edu; Marsha A Wilcox - marsha.wilcox@i3drugsafety.com; John S Witte - WitteJ@humgen.ucsf.edu; Qiong Yang - qyang@bu.edu; Andreas Ziegler - ziegler@imbs.uni-luebeck.de; Laura Almasy - almasy@sfbrgenetics.org; Jean W MacCluer - jean@sfbrgenetics.org * Corresponding author

from Genetic Analysis Workshop 15

St. Pete Beach, Florida, USA. II-I5 November 2006

Published: 18 December 2007

BMC Proceedings 2007, I(Suppl I):SI

This article is available from: http://www.biomedcentral.com/I753-656I/I/SI/SI

(C) 2007 Cordell et al; licensee BioMed Central Ltd.

This is an open access article distributed under the terms of the Creative Commons Attribution License (http://creativecommons.org/licenses/by/2.0), which permits unrestricted use, distribution, and reproduction in any medium, provided the original work is properly cited. 


\section{Preface}

This issue of BMC Proceedings contains the proceedings of Genetic Analysis Workshop (GAW) 15, which was held November 11-15, 2006, in St. Pete Beach, Florida, USA. The GAWs began in 1982 and are now held in even-numbered years. They provide a forum for investigators interested in identifying genetic effects on complex diseases to evaluate and compare novel and existing statistical methods. The purpose of these Workshops is to allow the comparison of statistical methodologies for genetic epidemiology using common, well described data sets. Prior to each GAW, topics are chosen, one or more existing data sets are selected, and a set of simulated data is created that permits investigation of current questions of broad interest in statistical genetics. These data are made available to any scientist who requests them, and their analyses of these data are presented at the Workshop. Participation in the Workshop is open to anyone who submits an analysis of one of these data sets, provides data, or participates in Workshop organization. More information about GAW, including details of upcoming Workshops, may be found at http://www.gaworkshop.org.

Three data sets (two empirical and one simulated) were distributed for GAW15, addressing two general classes of problems: 1) the genetics of expression, and 2) methods for dissection of complex traits. For the first time, one of the real data sets included RNA expression data from microarrays (Problem 1). Analyses of these data by GAW15 participants were focused primarily on expression data as quantitative traits in linkage and association scans and on methods for extracting additional information from the massively multivariate nature of the data set, which included literally thousands of quantitative traits. The second real data set (Problem 2) provided opportunities to address such methodological problems as separating multiple functional loci within a region of linkage or association, and linkage and association analyses of markers in the pseudoautosomal region of the $\mathrm{X}$ chromosome. The simulated data set (Problem 3) for GAW15 was based on the Problem 2 data set to allow participants to address complementary questions in a data set with a known genetic architecture. Here we provide a brief summary of the data sets; further details can be found in Cheung and Spielman [1], Amos et al. [2], and Miller et al. [3] in this issue.

The Problem 1 data set included microarray expression profiles originally investigated by Morley at el. [4]. Data were provided for 14 three-generation Centre d'Etude du Polymorphisme Humain (CEPH) Utah families (approximately 8 offspring per sibship and approximately 14 individuals per family). Phenotypes included expression level of genes in lymphoblastoid cells of these family members, obtained using the Affymetrix Human Focus
Arrays that contain probes for 8500 transcripts. Among these, Morley et al. [4] found greater variation among individuals than between replicate determinations on the same individual for 3554 expression phenotypes (expressed genes); these were provided to GAW15. For approximately 100 individuals, array hybridizations were performed in duplicate. The Affymetrix CEL files for all array hybridizations were provided to GAW15 participants. Genotypes for members of the 14 families were provided for 2882 autosomal and X-linked single-nucleotide polymorphisms (SNPs). The genotypes were generated by The SNP Consortium. This data set provided the opportunity to develop and apply methods for simultaneous analysis of a variety of related traits. Natural variation in gene expression is a new idea, and this collection is the first to provide such a large number of phenotypes in a family study.

The Problem 2 data set consisted of family- and population-based data from the North American Rheumatoid Arthritis Consortium study (NARAC) and from collaborators in Canada, France, and England. The goal of these studies is to understand the etiology of rheumatoid arthritis. It is highly likely that multiple interacting loci influence disease risk, as evidenced by the considerably higher recurrence risk for this disease to siblings as opposed to more distant relatives. The data provided by NARAC to GAW15 included 757 multiplex families genome scanned with microsatellites (511 families) and/or SNPs (746 families), candidate gene data for the PTPN22 locus from a study of 1519 controls and 1393 cases (and for additional candidate loci in a separate sample of 855 controls and 839 cases), dense genotyping data from a panel of 2300 SNPs for an approximately 10-kb region of chromosome 18q (genotyped on 460 cases and 460 controls), and further data on a number of quantitative phenotypes and clinical measures. The Canadian group provided data from 60 families that had been genotyped using the same Illumina platform used by NARAC as well as 79 families that were genotyped using an Affymetrix $100 \mathrm{~K}$ platform. The European Consortium on Rheumatoid Arthritis Families provided high-density microsatellite data from 88 families typed with 1089 microsatellite markers. The UK group provided microsatellite genome screen data from 174 families, of which 157 were also genotyped at 10,156 SNPs. A further set of 195 families genotyped at selected microsatellites was also provided.

The Problem 3 data set included 100 replicates of simulated data, modeled after the rheumatoid arthritis data set. Each replicate included 1500 nuclear families of size four (two parents and an affected sib pair (ASP)) and 2000 unrelated controls. Three sets of autosomal markers were generated: 1) a set of 730 microsatellite markers spaced on average $5 \mathrm{cM}$ apart; 2) a set of 9187 SNPs distributed on 
the genome to mimic a $10 \mathrm{~K}$ SNP chip set; and 3) a very dense map of 17820 SNPs on chromosome 6 (an average inter-marker spacing of $9586 \mathrm{bp}$ ). The data included map information, with lists of markers and their locations, and simulated family, marker, and phenotype/covariate data. "Answers" (the locations/effects of true causal loci and a description of the underlying generating model) were provided to GAW15 participants on request.

The availability of the GAW15 data was announced by email in the Spring of 2006, to the more than 2600 individuals on the GAW mailing list. A total of 179 groups requested GAW15 data. The Problem 1 data were requested by 133 groups, the Problem 2 data by 142 groups, and the Problem 3 data by 128 groups (with many groups requesting access to more than one data set). In the Summer of 2006, 252 contributed papers were received describing analyses of these data sets. A book and $\mathrm{CD}$ containing these contributions plus descriptions of the data sets were distributed to GAW15 participants.

The GAW15 participants included 350 individuals from 20 countries on four continents - Asia, Australia, Europe, North and South America. The 252 contributions submitted to GAW15 were organized into 17 presentation groups of 11 to 18 papers each, grouped based on common methodological themes. The 17 presentation groups were organized around the following themes: association analysis (Problem 1); association analysis (Problem 2); association analysis (Problem 3); combining linkage and association; data mining, neural networks, and gene networks (Problem 1); data mining, neural networks, and trees (Problems 2 and 3); gene $\times$ gene interaction; gene $\times$ environment interaction; linkage analysis (Problem 1); linkage analysis (Problems 2 and 3); model selection and Bayesian methods; multivariate analysis; candidate gene association; multistage designs; multiple testing and false discovery rate; processing and normalization of expression traits and their effect on analysis; and SNP selection, ancestry informative markers, and linkage disequilibrium between markers. For each presentation group, a group leader was chosen who had previous GAW experience. This person facilitated group discussion, organized the group's oral presentation to the general GAW meeting, and took the lead in writing the group summary paper to be published in Genetic Epidemiology.

Members of most presentation groups began interacting by email and/or conference call before GAW15, comparing and contrasting their approaches and results. Each presentation group also met at least once during the Workshop, where they continued their discussions and finalized a group presentation that was delivered to the full GAW15 audience during the general sessions. The group meetings were attended mostly by group partici- pants but were open to all GAW15 attendees. During poster sessions, 118 individual contributions were presented. There also was a special general session on "Novel Methods" at which four of the contributions (selected prior to GAW15 on the basis of the submitted papers) that had used or developed novel analytical approaches were highlighted and presented.

The 162 GAW contributions included in this issue of $B M C$ Proceedings are a subset of the 252 contributions presented at GAW15. All of these papers have been peer-reviewed and were selected on the basis of scientific merit. First come three papers that describe the data sets. These are followed by the 162 individual GAW15 contributions organized by presentation group, and alphabetically by first author within each group. Additionally, in a forthcoming supplement to the journal Genetic Epidemiology, a paper by each presentation group summarizes the contributions to that group and the lessons learned, comparing and contrasting contributions and describing their main themes and results. Overall, GAW15 generated many interesting discussions and some conclusions concerning appropriate approaches to the analysis of massively multivariate data, and methods for separating multiple functional loci within a region of linkage or association. These discussions also highlighted areas in which further methodological development is needed.

\section{Competing interests}

The author(s) declare that they have no competing interests.

\section{Acknowledgements}

The GAWs succeed because of the dedication of hundreds of individuals who help to select Workshop topics, provide real and simulated data, prepare and distribute data to participants, prepare Workshop contributions, organize the Workshop, lead presentation groups and chair sessions, write summary papers, review manuscripts, and edit the Workshop proceedings.

We are extremely grateful to the investigators who provide data for the Genetic Analysis Workshops for analysis by workshop participants. The workshops would not be possible without their generosity. Many investigators contributed data to GAWI5: Problem I: Drs. Vivian Cheung and Richard Spielman provided their microarray expression data from $\mathrm{CEPH}$ families. The generation of these data was supported by $\mathrm{NIH}$ grant HG002386 from the National Human Genome Research Institute (NHGRI). Problem 2: Rheumatoid arthritis data were provided by investigators in the US (Peter Gregersen, Elaine Remmers, Lindsey Criswell, Ann Begovich, Robert Plenge, Chris Amos, Wei V. Chen, Dan Kastner, Michael Seldin, Annette Lee), Canada (Katherine Siminovitch), the UK (Jane Worthington, Sally John, Neal Shephard), and France (François Cornelis). Dakai Zhu helped with organization of the data and Toi Soh assisted with preparation of the summary. The rheumatoid arthritis studies were supported by NIH grant AR44422, NIH contract NOI-AR-7-2232, funding from Genome Canada and Associations AFP, Polyarctique-Groupe Taitbout and Rhumatisme et Travail. Funding for UK researchers was provided by the Arthritis Research Campaign. Problem 3: The GAWI5 simulated data set was generated by Michael B. Miller in collaboration with Gregg R. Lind, Na Li, 
and Soon-Young Jang, with assistance from Octave developer John Eaton. Chris Amos was especially helpful in guiding choices in model selection. Support for generation of the simulated data was provided from $\mathrm{NIH}$ grants 5R0I-HL049609-I4, IROI-AG02 I9I7-0IAI, the University of Minnesota, and the Minnesota Supercomputing Institute.

The GAWI5 contributions were organized by theme into presentation groups. Seventeen people generously volunteered for the difficult task of group leader, which involved initiating interactions among group members before GAWI5, leading group meetings at GAWI5, organizing summary presentations for the larger GAWI 5 audience, and taking responsibility for the preparation of a summary paper for Genetic Epidemiology. Their efforts deserve special recognition. We are grateful to the following people who led the group discussions and preparation of summary presentations (in group numerical order): John Witte, Marsha Wilcox, Heike Bickeböller, L. Adrienne Cupples, Cathy Falk, Andreas Ziegler, Veronica Vieland, Rob Culverhouse, Ellen Wijsman, Saurabh Ghosh, Duncan Thomas, Joseph Beyene, Mariza de Andrade, Beth Hauser, Lisa Martin, Maria Martinez, and Josée Dupuis.

Useful comments and criticisms of the papers in this volume were provided by I 45 scientific reviewers: Goncalo Abecasis, Adeniyi Adewale, Alexandre Alcais, Andrew Allen, Chris Amos, Allison Ashley-Koch, Elizabeth Atkinson, Christy Avery, Michael Badzioch, Agnes Baffoe-Bonnie, Joan BaileyWilson, M. Michael Barmada, Jill Barnholtz-Sloan, Jenny Barrett, Terri Beaty, Lars Beckmann, Joanna Biernacka, Tim Bishop, Mike Boehnke, Stefan Böhringer, Catherine Bonaïti-Pellié, Catherine Bourgain, Alfonso Buil, Shelley Bull, Paul Burton, Nicola Camp, Rita Cantor, Jenny Chang-Claude, WeiMin Chen, Andy Collins, Michael Conneally, Nancy Cox, Florence Demenais, Anita DeStefano, Marcella Devoto, Guoqing Diao, Irina Dinu, MarieHelene Dizier, Marie-Pierre Dube, Frank Dudbridge, Priya Duggal, Ravi Duggirala, Jeanette Eckel-Passow, Howard Edenberg, Sarah Ennis, Carol Etzel, Dani Fallin, Cathy Fann, Christine Fischer, Nora Franceschini, Brooke Fridley, France Gagnon, Chad Garner, Emmanuelle Génin, Rodney Go, David Goldgar, Lynn Goldin, Alisa Goldstein, Derek Gordon, Harald Göring, Celia Greenwood, Courtney Gray-McGuire, Fangyi Gu, Chao-Yu Guo, Jonathan Haines, Robert Hanson, Sandy Hasstedt, Simon Heath, Tony Hinrichs, Peter Holmans, Torsten Hothorn, Jeanine Houwing-Duistermaat, Yifan Huang, Cristina Justice, Candace Kammerer, Xiayi Ke, Abbas Khalili, Terri King, Andre Kleensang, Alison Klein, Inke König, Peter Kraft, Carl Langefeld, Martin Larson, Chun Li, Jing Li, Mingyao Li, Wentian Li, Shili Lin, Kathy Lunetta, Brion Maher, Jim Malley, Nik Maniatis, Jeanette McCarthy, Nancy Mendell, Chantal Merette, Brackie Mitchell, Richard Morris, Nandita Mukhopadhyay, Betram Muller-Myhsok, Deborah Myers, Rosalind Neuman, Kristen Nicodemus, Dahlia Nielsen, Nora Nock, Jeff O'Connell, Jurg Ott, Grier Page, V. Shane Pankratz, Charalampos Papachristou, George Papanicolaou, Andrew Paterson, Roy Perlis, Ruth Pfeiffer, Silvano Presciuttini, Elizabeth Pugh, Dajun Qian, John Rice, Steve Rich, Marylyn Ritchie, Santiago Rodriguez, Glen Satten, Mike Schmidt, Svati Shah, Sanjay Shete, Kim Siegmund, Janet Sinsheimer, Susan Slager, Anne Spence, Hans Stassen, Cathy Stein, Karen T. Cuenco, Dawn Teare, Alun Thomas, Elizabeth Thompson, David Tregouet, Sita Vermeulen, Kai Wang, Shuang Wang, Michael Weale, Jessica Woo, Qiong Yang, Yutaka Yasui, Robert Yu, and Xiaofeng Zhu. We are grateful for their contributions.

Since GAW7 in 1991, Vanessa Olmo has had major responsibility for all aspects of Workshop organization. Over the years, as the Workshops have increased in size and complexity, she has taken on greatly increased responsibilities. She has primary responsibility for Workshop logistics, including interaction with participants, organizers, editors, and publisher; data distribution; site selection and liaison with local organizers; maintenance of the GAW web site and mailing list; and preparation of the proceedings. The
GAWs could not succeed without her commitment and her enthusiasm. We also thank Selina Flores who helped with data distribution, communications with participants, and preparation of the pre-GAW volume; and Richard Polich, Tom Dyer, Laura Almasy, Linda Freeman-Shade, and Gerry Vest, who worked on preparing the data for distribution. As for past GAWs, April Hopstetter, Director of Technical Publications and Printing at the Southwest Foundation for Biomedical Research, assisted with editing of the GAWI 5 proceedings, while Maria Messenger and Malinda Mann typeset the articles. Rene Sandoval and Rudy Sandoval were responsible for putting together the final pre-GAW book.

Local arrangements for GAWI5 required countless hours of planning and organization. We are grateful to Tom Sellers, and the local organizer, Vicki Slusher, as well as volunteers Jill Barnholtz-Sloan, Yifan Huang, Virna Dapic and Alison Fay for their efforts to assure a successful GAW. Funding for scholarships to postdoctoral fellows and graduate students to help defray their expenses in attending GAWI5 were provided by NIGMS, Celera Diagnostics, Illumina, Inc., and Nature Publishing Group. We are grateful for their generosity.

The GAW Advisory Committee, which has a rotating membership, has overall responsibility for long-term planning for the GAWs. Its membership at the time of GAWI5 included Laura Almasy, Joan Bailey-Wilson, Heike Bickeböller, Ingrid Borecki, Françoise Clerget-Darpoux, Heather Cordell, Lynn Goldin, Jean MacCluer (chairman), Duncan Thomas, John Witte, and Andreas Ziegler.

Continuous funding for the GAWs has been provided since 1982 by the National Institute of General Medical Sciences (NIGMS), through grant ROI GM3I575 to Jean MacCluer. We wish to thank Dr. Richard Anderson of NIGMS for his interest in GAW and for his efforts as Program Director for the GAW grant. We are particularly grateful to Irene Eckstrand of NIGMS for her enthusiasm and interest in the GAWs since they were first envisioned in 1981. The GAWs would not be possible without the support of Drs. Eckstrand and Anderson and NIGMS.

Finally, we wish to express our gratitude to the GAW participants, without whose ongoing, enthusiastic support the GAWs could not have enjoyed their continuing success.

This article has been published as part of BMC Proceedings Volume I Supplement 1, 2007: Genetic Analysis Workshop 15: Gene Expression Analysis and Approaches to Detecting Multiple Functional Loci. The full contents of the supplement are available online at http://www.biomedcentral.com/ $|753-656| / \mid$ ? issue=SI.

\section{References}

I. Cheung VG, Spielman RS: Data for Genetic Analysis Workshop I5 (GAW I5), problem I: Genetics of gene expression variation in humans. BMC Proc 2007, I(SuppI I):S2.

2. Amos Cl, Chen WV, Remmers E, Siminivitch KA, Seldin MF, Criswell LA, Lee AT, John S, Shephard ND, Worthington J, Cornelis F, Plenge RM, Begovich AB, Dyer TD, Kastner DL, Gregersen PK: Data for Genetic Analysis Workshop (GAW) 15 problem 2, genetic causes of rheumatoid arthritis and associated traits. BMC Proc 2007, I (SuppI I):S3.

3. Miller M, Lind GR, Li N, Jang S-Y: Genetic Analysis Workshop I5: Simulation of a complex genetic model for rheumatoid arthritis in nuclear families including a dense SNP map with linkage disequilibrium between marker loci and trait loci. BMC Proc 2007, I(Suppl I):S4.

4. Morley M, Molony CM, Weber T, Devlin JL, Ewens KG, Spielman RS, Cheung VG: Genetic analysis of genome-wide variation in human gene expression. Nature 2004, 430:743-747. 